\title{
The Implementation of Oilfield Operation Simulation Examination System Client Based on Mobile Device
}

\author{
Ya Zhao ${ }^{1, *}$, Lina Sun ${ }^{1}$, Shengyi $\mathrm{Lv}^{2}$ and Lin $\operatorname{Lin}^{1}$ \\ ${ }^{1}$ Institute of Computer \& Information Technology, North-East Petroleum University, Daqing Heilongjiang 163318, China; \\ ${ }^{2}$ Institute of Higher Education, North-East Petroleum University, Daqing Heilongjiang 163318, China; \\ ${ }^{*}$ Corresponding author
}

\begin{abstract}
This paper aims to research and develop the oilfield operation simulation examination system, which can run on the mobile devices without network. According to the system needs, the paper analyzes the functional requirements of the server and the client; designs the functions according to the client needs; researches some key technologies; describes the concrete realization process of the functions, finally completes the simulation examination system client which can run on the mobile device fluently. The system obtains good effect after being used in oilfield operation, can help the workers be familiar with the operation process before going to work in 5 minutes and help them avoid the generation of operation accidents effectively, receives recognition from the oilfield company, has the potential of being promoted to other oilfields.
\end{abstract}

Keywords-simulation examination system; unity 3D; mobile device; oilfield operation

\section{INTRODUCTION}

Safety is the permanent goal of oilfield operation. In order to reduce the incidence of accidents, oilfield workers need be trained and tested before working. The traditional training methods take the mode of unified centralized test, which need concentrated and long time, cost much manpower, material and resources. With the emergency of virtual reality technology, various training and examination simulation system about oilfield operation arise in this environment. These systems save the training time and training consumption. The characters of virtual reality ensure the real sense of experience in operation, enhance the training efficiency.

With the promoting of the importance degree in oilfield operation safety, the leaders think out the idea that making use of the simulation system to test the workers before beginning work in 5 minutes. If the workers pass the examination, they can go to work, if they can't pass it, they can't go to work. The purpose is to minimize the possibility of accident in oilfield operation. The simulation systems described before usually to be used by these steps: logging in PC machines, connecting the server through the network and then use it. This method has limit in fieldwork of oilfield operation.

Previously mentioned, the paper aims to research the oilfield operation simulation examination system which can run on the intelligent mobile devices without network. The system described in this paper runs on the intelligent mobile devices as client. The previous data required in system can be downloaded from the data server to local devices. The later data gotten from the examination can be stored in local devices temporarily, be transferred to the data server through network, facilitate the oilfield managers supervise the examination situation before beginning work of the workers.

\section{SySTEM FUNCTIONAL REQUIREMENTS AND DESIGN}

\section{A. Functional Requirement of Server}

The normal operation of the client can't be separated from the server[1]. According to the system function analysis, the server mainly provides data support and communication support for the client.

\section{1) Database need}

When the user running the virtual simulation examination system on the mobile devices, he needs download the related data information from the database, including: user role information, simulation examination operation module related information, operation process information of each operation module, etc.

\section{2) Data communication need}

In this system, the data are carried out in the form of a data packet through the data communication protocol between the client and the server. So we need to establish a set of accurate and complete data communication protocol, high performance and compact data interchange format matching the protocol.

\section{B. The Functional Need and Design of the System Client}

\section{1) The functional need of the system client}

The virtual simulation examination system client is the core function of the whole system. On the one hand, it is the realization of data communication function; on the other hand, it is the realization of system virtual examination function.

The data communication function between system client and server is realized by the combination of them.

During the implementation of each operation module examination function, the system client needs to construct 3d object models and character models of each operation module, integrate the scene and optimize it, write control logic to realize the examination function of the system. 


\section{2) The design of system client}

According to the functional requirements analysis of the client, the design of the client is divided into three levels: presentation level (includes 3D modeling, scene integration and optimization), logic level (the examination function writing of the control logic scripts), data communication level (the data communication between the client and the server). In addition, in view of the various independent examination operation module included in the system client, it also needs to carry on the function structure of the module content and the design of the examination process.

\section{The KeY TEChNOLOgIES UsED IN THE SysteM}

\section{A. Dynamic Display Technology of the Model}

Due to the limitation of hardware, the running rate of mobile devices is limited. In order to improve the running speed of the system, the dynamic display technology of the model is adopted.

During the system running, each user assessed has a camera which simulating his viewpoint, the observation scope of the viewpoint in the examination is mainly focused on this camera. Dynamic display technology of the model obtains the camera's position information through writing class, gets the position information of other models and characters in the scene, and gives a threshold value. Through calculating the distance between these models and the camera on behalf of user viewpoint, comparing the distance with the threshold, rendering the models whose distance in the threshold area, this can effectively improve the running rate of the system. The decision method diagram of dynamic display determination method of the model is shown in figure 1 .

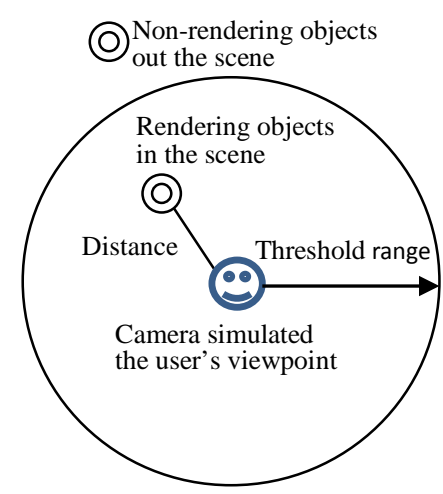

FIGURE I. DECISION METHOD DIAGRAM OF DYNAMIC DISPLAY DETERMINATION METHOD OF THE MODEL

\section{B. Automatic Switch Control of the Camera Viewpoints}

The virtual simulation examination system can't separate from the interaction of viewpoints. Users can observe the objects in virtual scene correctly through viewpoint. In the process of examining the system operation modules, the user' $s$ viewpoint needs to change with the transform of the positon of the tools or characters in the scene, so to ensure the correctness of the object observed. In the virtual scene, the user's viewpoint is simulated through the camera. it can simulate the change of the user's viewpoint by adjusting the position and the angle of the system camera.

Considering the accuracy switch and the fast location of viewpoint, the system adopts a general automatic camera viewpoint switching method: Set the parent object for the camera. So when the user's viewpoint needs to switch, change the camera's parent object into the one which nears the viewpoint to determine the camera's position information, adjust the camera angle to achieve the best viewing angle, and achieve the purpose of changing the viewpoint accurately to observe the objects.

\section{The Virtual Character's Automatic Pathfinding}

The virtual character's automatic pathfinding is an inevitable problem to solve in 3D game and virtual simulation system. The typical path planning algorithm includes Dijkstra and $\mathrm{A}^{*}$ algorithm, etc. [3].

The system need to realize the automatic pathfinding of the virtual character. The realization of this function mainly uses a kind of optimization algorithm similar to $\mathrm{A}^{*}$ algorithm provided by Unity. This algorithm is suitable for the environment with static obstacles and constant working space. The algorithm's path planning can be divided into two steps: coordinate based modeling of the scene and path searching. Unity3D simplifies the complex structure of the system scene into a grid with some information by using NavMesh( navigation mesh) component, that is, does coordinate based modeling of the scene. Then, it realizes the automatic pathfinding of virtual characters by a series of calculations on the basis of these grids, that is, does path searching for virtual characters.

\section{Screen Adaptation}

The system realized in this paper runs on the mobile devices. Limited by the manufacturer, the mobile devices in the market have different resolutions, there is no uniform standard. Therefore, if we don't consider the resolution of the device, the system's UI in different mobile devices will be compressed or stretched, lose the original beauty of the interface. In order to solve this problem, we should consider the interface location problem in different resolution of the mobile devices fully, that is, the problem of screen adaptation. The specific implementation process is shown in Figure 2.

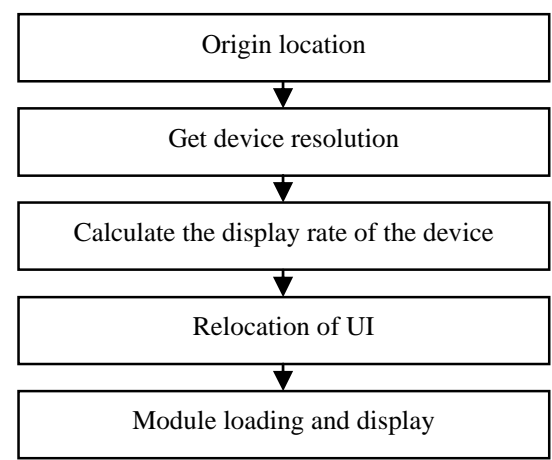

FIGURE II. THE IMPLEMENTATION PROCESS OF SCREEN ADAPTATION 
Since the choice of specific operation module, the first step is doing the original location of UI in the mobile device which the module runs on, we set the origin coordinate in the upper left corner of the mobile device in this system; the second step is acquiring the resolution of the mobile device, calculating the display scale of the device screen according to its resolution, so to carry out the UI relocation; after all of these the last step is to load and display the module. This method can ensure that the system can be displayed and used normally in different mobile devices having different resolutions.

\section{THE REALIZATION OF THE SYSTEM}

\section{A. Object and Character Modeling, Animation Production}

The realization of virtual simulation system is based on 3D model, including object model and role model.3D model construction is a method that transforms the objects and attributes in the real world into the expression of the machine world[4].

\section{1) Material arrangement}

After the investigation of the information including the scene of specific oilfield operations, the equipment and tools involved in the operations, personnel involved in the operations, etc., we should classify the information into object classes and character classes. Object classes: outline the operation field layout, collect the parameters of the equipment and tools' shape photos, specific size, internal and external structure, and take as many pictures for texture mapping. Character classes: explicit the character's external features, includes the clothes and portable tools of different workers in this system.

\section{2) Model construction and beautification}

The principle of modeling is: try to using as less faces and material balls as possible to keep the reality of the model.

Object class models: the objects contained in the system are constructed by the methods of basic modeling, composite modeling, Nurbs modeling, editor editing and modifying, etc., set reasonable texture mapping.

Character class models: we construct the virtual character's shape by the method of geometric modeling, construct the skeleton to support the virtual character's motion, at last make character skinning by setting texture mapping. All of these ensure the reality of the character in our system. The following figure shows some models in our system.
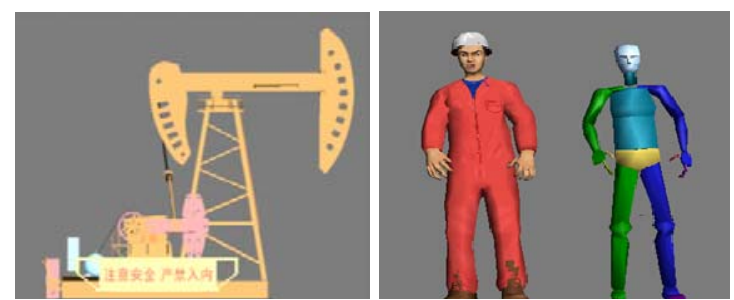

FIGURE III. THE MODELS OF AN OIL PUMPING MACHINE AND A VIRTUAL CHARACTER

\section{3) Animation production}

Some of the object's own periodic motion in the system, such as the reciprocating motion of the pumping machine and the fixed action of virtual character, are hardly realized in Unity3D and the effect is not good, but they are relatively simple to achieve in Max. Unity3D provides the component which can quote Max animation directly. Therefore, we can make the object motion and the character action to animation clips directly in Max, export and quote them in Unity3D directly.

\section{B. Scene Integration and Optimization}

Each operation module contained in this system including different equipment and tools. Therefore, we should complete the integration of the scene and construct the virtual operation scene consistent with the real one.

In order to achieve physical simulation of the integrated virtual operation scene, further landscaping is needed in the light efficiency and the surrounding environment. These includes: the natural light, the real light shadow and the natural surrounding environment.

\section{The Realization of Control Logic}

The examination function of each operation module is mainly realized through writing control logic. The system logic functions include the following parts: data communication logic, examination data logic, user login logic, scene loading logic, operation response logic, performance management logic. The trend graph of control logic is shown in Figure 4.

Data communication logic mainly completes the data communication between the client and server, including the development of data communication protocol and data communication realization.

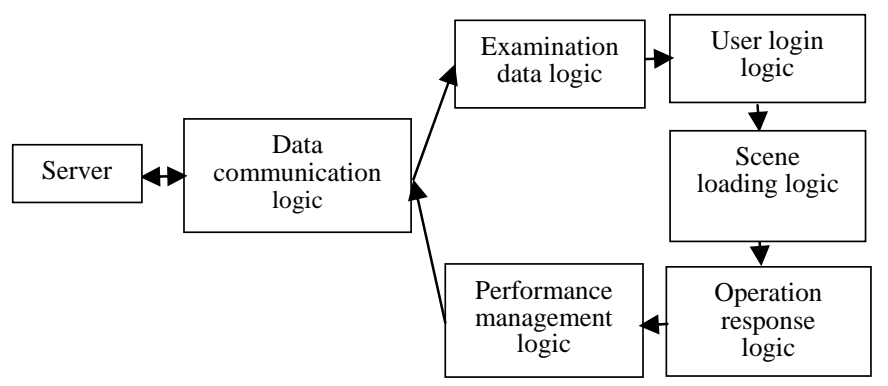

FIGURE IV. THE TREND GRAPH OF CONTROL LOGIC

Examination data logic requires user information and operation information from the server through data communication logic to the mobile device client and stores locally.

User login logic is used to realize the login by character of the oilfield operation workers in each examination module, so as to extract the examination operation process information of the character in this operation module. 
Scene load logic loads the virtual scene of the operation module which the user entered after the user login. The simulation examination carries on in this virtual scene.

Operation response logic responds to the user's examination operation: playing the process operation content, enabling the operation tool, and changing the equipment's status, etc., carries out the operation process show of the specific operation effectively and orderly.

Performance management logic manages the results during the process of examination, including records of each examination operation process completed or not, each examination process' scores, the user's total score when the examination is completed. The information will be stored in the mobile devices as a data file after recorded, so that to be stored in the server database through data communication logic under the network.

\section{The Realization of Data Communication between the Client and Server}

\section{1) The definition of communication protocol}

Communication protocol is defined as a certain data format and identification code (request identifier and response identifier), which is in the prior agreement between the client and server. When the client and server do data communication, first they encapsulate the communication data in this format, and transmit it via network protocol. They received data packets are analyzed in accordance with the agreement. They restore the transmission data, so as to complete the data communication between the two aspects. In the definition of communication protocols, it is necessary to consider sufficiently of the efficiency and security of the communication process[5]. So the package structure, the encoding and decoding format of the data contained in the protocol are very important.

The communication protocol defined in this system is an interactive protocol based on data packets, which transmits the data via TCP/IP network protocol. The format of the data package is defined as follows: identification code + whole package length + package type + packet header + package body.

\section{2) The preparation and serialization of protocol data file}

Protocol Buffers is a mixed language data standard for Google company, and it is a kind of portable and efficient structured data storage format, can be used to sequence structured data[6-7]. It is used to carry out the serialization of the communication protocol and the realization of the communication, the specific implementation process is as follows:

- Write the data definition files in Notepad, then save the file by selecting the encoding for UTF-8 and suffix for the .proto.

- Translate the stored data definition files using transformation tool ProtoGen into data access class files suffix for the .cs.
- Compile the data access calss file into a dynamic link library to call.

\section{3) The realization of data communication}

According to the definition method of data communication, two data definition files are written in the system:

- Request data definition file: this file is used by the client to initiate a request to the server.

- Response data definition file: this file is used by the server to respond to the client.

When the data communication function is enabled, the request instruction and data packets are sent to the server by the client. After receiving the package, the server analyzes the package first, clears the operation need of the client, and verifies the legitimacy of the requested operation, implements of the requested operation. Then the server sends the reply instruction and the result data packet to the client side. After receiving the packet, the client analyzes the package first, gets the data they need, and executes the corresponding operation. The specific communication process is shown in Figure 5.

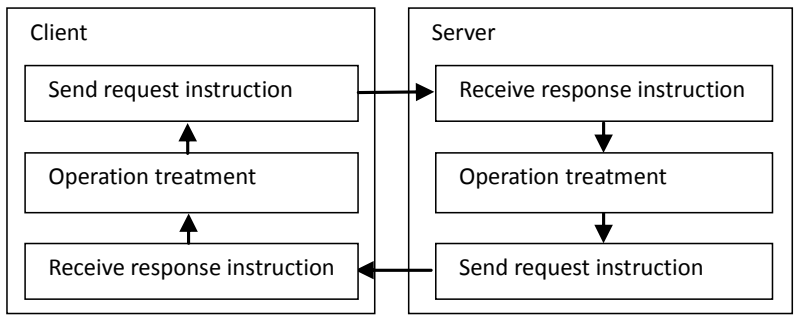

FIGURE V. THE DATA COMMUNICATION PROCESS

\section{CONCLUSION}

The design and implementation of the client running on the mobile device is discussed in this paper. The system can run stably on mobile devices, fully meet the need of the oilfield worker examined in 5 minutes before beginning work as well as can go to work after passing the examination. It can help the oilfield worker fully familiar with the operation process before beginning work, effectively avoid the accidents of operation, receive recognition from the oilfield operation company, has the potential of being promoted to other oilfields.

\section{ACKNOWLEDGMENT}

This work is supported by the Education Science Key Research Project of Heilongjiang Province \# GJB1215019, the Graduate Education Innovation Project of Heilongjiang Province \#JGXM-HLJ-2015111, the National Natural Science Foundation of Chain Project \#61402099.

\section{REFERENCES}

[1] Z. T. Su, S. K. Shi, J. H. Li. "Research on development framework for mobile games", Computer Engineering and Design. vol. 21, pp. 16311634, July 2010. 
[2] B. Y. Li, C. X. Zhao, H. F. Zhang. "New Method of Fast Laser Scan Ranger Simulation in ALV System”, Journal of System Simulation. vol. 21, pp. 5075-5079, August 2009.

[3] Z. M. Tang, C. X. Zhao, H. J. Sun, J. F. Lu, J. Y. Yang. "The Information Fusion and Trejectory Planning System for Autonomous Ground Vehicle", Journal of Nanjing University of Science and Technology. vol. 27, pp. 6-10, Janury 2003.

[4] B. Qu, Y. Zhao, Q. Zhao. "Design and Implementation of a Virtual Home Ramble System Based on Virtools". Computer Engineering \& Science. vol. 31, pp. 130-133, December 2009.

[5] C. H. Wang, Research and Design of EPOLL-based the Network Game Server Communication Structure. Dalian Maritime University, pp. 44-46, Dalian(2012).

[6] J. B. Ren, Design and implementation of client for phone game based on the Unity3D. Beijing Jiaotong University, pp. 5-7, Beijing(2013).

[7] J. F. Chen, Research and Implementation of a cross-platform mobile online game based on the Unity3D game engine. Zhongshan University. pp.8-14, Zhongshan(2013) 